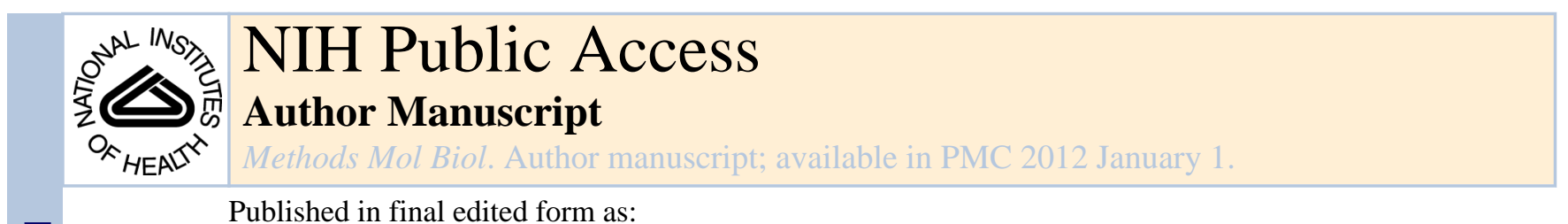

Published in final edited form as:

Methods Mol Biol. 2012 ; 827: 215-234. doi:10.1007/978-1-61779-442-1_15.

\title{
Multiplex Imaging of Rho Family GTPase Activities in Living Cells
}

\author{
Désirée Spiering and Louis Hodgson
}

\begin{abstract}
Here, we provide procedures for imaging the Rho GTPase biosensors in both single and multiplex acquisition modes. The multiplex approach enables the direct visualization of two biosensor readouts from a single living cell. Here, we take as an example a combination of the RhoA biosensor based on a CFP/YFP FRET modality and the Cdc42 biosensor based on organic dyes that change fluorescence as a function of the local solvent polarity. We list the required optical components as well as cellular manipulation techniques necessary to successfully image these two ratiometric biosensors in a single living cell.
\end{abstract}

\section{Keywords}

Rho GTPase; Dynamics; Live-cell imaging; FRET; Biosensors; Multiplex

\section{Introduction}

The fluorescent biosensors for detecting the activation of the Rho family of p21 small GTPases have opened a new window into the spatiotemporal regulation of these important molecular switches in living cells (1-7). The ability to observe the effector-binding events of these proteins in their native environment has significantly changed the way in which we understand the biology of Rho GTPases (1-5). While the early imaging attempts of these sensors were limited to the single-mode observations wherein only one biosensor was imaged in a single living cell at a time, we have now capabilities to observe two distinct Rho GTPase activities in a single living cell (4). This latter approach has enabled the direct characterization of the interrelationships between the activation states of different Rho GTPases to better understand the balance and coordination of Rho GTPase activations that govern the leading edge dynamics (4). Here, we provide methods for both the single-mode and the multiplex-mode imaging approaches.

\subsection{General Considerations for the Use of Rho GTPase Biosensors}

The biosensors based on CFP/YFP fluorescence resonance energy transfer (FRET) such as the Rho GTPase biosensors (3) or those that are based on solvent-sensitive dyes $(2,8)$ require ratiometric calculations. Methods for observing single protein activity in living cells using these types of biosensors are described in detail elsewhere (9). Here, we summarize some key issues, specific caveats, and pitfalls encountered during this mode of imaging. There are several major considerations: (1) expression levels of the biosensor and the dominant negative effect; (2) motion artifacts; (3) lateral chromatic aberrations within the field of view and dual-camera alignment; and (4) general imaging considerations for optimal live-cell microscopy. These issues are addressed in the following sections. 
The expression level of the genetically encoded biosensors requires careful control in order to maintain a minimal level of biosensors needed to obtain a sufficient signal-to-noise ratio (SNR) during imaging. Typically, the SNR for the widefield epifluorescence mode we employ is 2:1 at the dimmest part of the cell periphery compared to the background fluorescence signals outside of the cell (9). We have found that in order to achieve this SNR, we must optimize the microscope optics by custom designing the bandpass filters and dichromatic mirrors. The specifications of the microscope optics that we employ for this mode of imaging are shown in Table 1. Using these custom components, we are able to maintain the biosensor expression levels at 20-30\% of the endogenous material (see Fig. 1) (4) and obtain sufficient SNR during imaging. To achieve these low levels of expressions, we routinely utilize an inducible gene expression system (tet-OFF) coupled to a retroviral transduction system where we can tightly regulate the induction levels of biosensors by titrating the amount of doxycyclin $(3,10,11)$, and also to control the relative copy numbers of the biosensor cDNA incorporated into the genome by optimizing the number of retroviral transduction cycles.

\subsection{General Considerations for the Single-Mode Imaging of Rho GTPase Biosensors}

The widefield imaging of live cells expressing the genetically encoded biosensor can be performed in one of two ways. The Sequential Imaging mode utilizes a single camera attached to the microscope imaging port via a filterwheel which can be used to switch the emission filters between the CFP and YFP FRET emissions. Two images of the biosensor are taken sequentially and later ratiometric calculations are performed. The major advantage of this approach is the simplicity of the hardware design where only a single high-sensitivity cooled charge-coupled device (CCD) camera is needed (see Fig. 2). A minor advantage could be that because a single CCD is used, it will be highly unlikely for any significant image misalignment to be present in the two fields of views acquired. However, the lateral chromatic aberration will be present, which will depend on the extent of the wavelength differences of the two acquisition wavelengths and on the relative location of the placement of the CCD within the field of view. Furthermore, because the filterwheel is normally positioned within the focused optical space, the differences in the thicknesses of the emission bandpass filters as well as their slight imperfections in the mounting of these filters could cause misalignments and defocusing effects at the image plane. These latter issues can be fully corrected via the calibration and computational correction methods developed previously (9), along with the use of a priori determined focus offsets for each of the channels. One of the major drawbacks of the sequential mode of acquisition is that, because it takes a finite amount of time to switch between the two emission wavelengths (together with the appropriate exposure times at each wavelength), the cell in the field of view could move in between the two sequential acquisitions. This will produce "motion artifacts" during the ratiometric calculations $(2,9)$, and will make the interpretation of the resulting ratiometric data set difficult. Approaches to roughly estimate the extent of such motion artifacts are described elsewhere $(2,9)$. In order to sidestep this critical problem, it is possible to set up two identical cameras for a simultaneous image acquisition. This requires an additional beam-splitter in the light path in place of the filterwheel in the emission optical train as well as a second identical CCD camera (see Fig. 3). The advantage of this approach is that it removes the motion artifacts through eliminating the need to switch the wavelengths. However, because the alignment of the cameras and the beam-splitter mirrors are not always perfect, it introduces additional sources of image misalignments including the field shear or otherwise nonlinear displacements in the field of view. These issues are corrected using a priori calibration and morphing-based computational image matching technologies allowing to register the two ratiometric images to within one-twentieth of a pixel accuracy (9). 


\subsection{General Considerations for the Multiplex-Mode Imaging of Rho GTPase Biosensors}

Using a combination of two biosensor systems with compatible fluorescence characteristics, it is now possible to perform the widefield imaging of two protein activities in single living cells $(4,12)$. This necessitates separations of four wavelengths for two ratiometric determinations of protein activities. One previous approach was to use a combination of a CFP/YFP FRET biosensor and another biosensor that was based on organic dyes (4). In this approach, dye fluorescence wavelengths and their placements on the biosensor molecule were carefully adjusted to be complementary to the genetically encoded biosensor in a single living cell. More recently, an approach was developed where a set of two genetically encoded biosensors (one using CFP/YFP FRET, another using the monomeric orange fluorescent protein 2 and monomeric Cherry) have been used in single living cells (12). However, this work, while enabling the measurements of two distinct readouts, utilized one biosensor that was intracellular and another as an extracellular expressed protein, segregating the compartments occupied by these two sensors, making the separation of wavelengths simpler (12). Naturally, if measurements of two protein activities from components that existed in the same compartment (such as the case of many Rho GTPases) are required, separation of fluorescence wavelengths is more challenging using this latter approach.

The optical system that enables separation of four wavelengths is shown in Fig. 4. The associated bandpass filters and dichromatic mirror specifications are shown in Table 2. In this specific configuration, the system is capable of imaging CFP/YFP FRET together with the dye-based biosensor utilizing the ratiometric dye combination of mero87 (13) and a farred dye, Atto-700-NHS with an excitation/emission wavelengths of 681/713 nm. Here, we will focus our discussions on the RhoA biosensor based on CFP/YFP FRET (3) and a modified version of the MeroCBD biosensor for detecting the activity of endogenous Cdc42 GTPase $(2,4)$.

\section{Materials}

\subsection{Cell Culture, Transfection, and Viral Transduction}

1. Cell lines: GP2-293 (Clontech) and mouse embryonic fibroblasts (MEF) (Clontech).

2. DMEM ( $4.5 \mathrm{~g} / \mathrm{ml}$ glucose with $110 \mathrm{mg} / \mathrm{L}$ sodium pyruvate; Mediatech) supplemented with $10 \%$ fetal calf serum (FCS), penicillin (100 IU)/streptomycin $(100 \mu \mathrm{g} / \mathrm{ml}$ ), and $2 \mathrm{mM}$ Glutamax (Invitrogen).

3. Opti-MEM (Invitrogen).

4. Trypsin (Mediatech).

5. Plasmids: $p$ Retro-X-tet-OFF system including the pRetro-X-Tight-Pur or the pRetro-X-Tight-Hygro backbone system (Clontech). The biosensor cDNA is restricted from the cloning vector and ligated into the multiple cloning site of the pRetro-X backbone at the NotI restriction site. Both the vector backbone and the insert must be blunt-ended using Klenow end-filling. The viral expression constructs for the RhoA bio-sensor can be obtained from Addgene (http://www.addgene.org) in the previous generation format (pBabe-sin-puro-tetCMV-RhoA Biosensor) or the current configuration in the pRetro-X system by writing to the authors.

6. Lipofectamine 2000 reagent (Invitrogen). 
7. DPBS, calcium and magnesium free (Mediatech): $0.2 \mathrm{~g} / \mathrm{L} \mathrm{KCl} ; 0.2 \mathrm{~g} / \mathrm{L} \mathrm{KH}_{2} \mathrm{PO}_{4} ; 8$ $\mathrm{g} / \mathrm{L} \mathrm{NaCl} ; 1.15 \mathrm{~g} / \mathrm{L} \mathrm{Na}_{2} \mathrm{HPO}_{4}$ (anhydrous).

8. Poly-L-lysine (Sigma P4707) diluted 1:10 in DPBS. For coating, treat plates with this solution for $5 \mathrm{~min}$ at $24^{\circ} \mathrm{C}$ and aspirate prior to plating cells.

9. Fibronectin (Sigma) diluted in DPBS at a final concentration of $10 \mu \mathrm{g} / \mathrm{ml}$. For coating, treat coverslips with this solution for $1 \mathrm{~h}$ at $24^{\circ} \mathrm{C}$ and aspirate prior to plating cells.

10. Retro-X concentrator (Clontech).

11. Polybrene (Sigma), $8 \mu \mathrm{g} / \mu$ l stock.

12. Antibiotics: G-418/neomycin ( $100 \mu \mathrm{g} / \mu \mathrm{l}$ stock), puromycin ( $10 \mu \mathrm{g} / \mu \mathrm{l}$ stock), hygromycin (50 $\mu \mathrm{g} / \mu \mathrm{l}$ stock), and doxycyclin ( $10 \mu \mathrm{g} / \mathrm{ml} \mathrm{stock})$.

13. Beckman Coulter MoFlo XDP FACS system.

\subsection{Dye Labeling of the Multiplex-Comptible Biosensor for Cdc42}

1. Escherichia coli strain expressing a Cdc42-binding domain fused to maltosebinding protein (CBD-MBP) (available from the author's laboratory).

2. Atto-700-NHS-succinimidylester (Sigma).

3. Mero87-Iodoacetamide (13). This reactive dye is available from the Hahn laboratory; University of North Carolina at Chapel Hill.

4. Centrifugal concentrators (Amicon Ultra $10 \mathrm{kDa}$ cut-off; Millipore).

5. Sephadex G-15 (Sigma).

6. $50 \mathrm{mM} \mathrm{NaH}_{2} \mathrm{PO}_{4}$ at $\mathrm{pH} 7.5$ and 5.5.

7. 2-Mercaptoethanol.

8. $1.5 \mathrm{M}$ Hydroxylamine (Sigma) at $\mathrm{pH}$ 5.5, prepared fresh.

\subsection{Imaging}

1. Refer to Tables 1 and 2 for the required optical filters and mirrors for the imaging setup. The placement of the CCD cameras for the different imaging modes is shown in Figs. 2-4.

2. Custom-made chamber for imaging (see Fig. 5). This chamber is designed to be airtight and is temperature regulated to $37^{\circ} \mathrm{C}$ when used in combination with a temperature regulator (QE-2; Warner Instruments).

3. 25-mm Round coverslips (\# 1.5; Warner Instruments).

4. Imaging medium: $6 \mathrm{ml}$ of Ham's F-12K without phenol-red (Biosource) (see Note 1) is warmed to $37^{\circ} \mathrm{C}$ to release any dissolved gases. Argon gas is then bubbled into the medium for $1 \mathrm{~min}$ to displace the oxygen. FCS is added at $2 \%$ and the medium is aliquoted into 2-ml tubes together with Oxyfluor reagent (Oxyrase) at 1:100 dilution and with $5 \mathrm{mM}$ DL-lactate. The mixture is then incubated at $37^{\circ} \mathrm{C}$ for $1 \mathrm{~h}$ and spun for $1 \mathrm{~min}$ at $24^{\circ} \mathrm{C}, 20,000 \times g$ to remove any debris from the Oxyfluor treatment prior to imaging (see Note 2 ).

\footnotetext{
${ }^{1}$ This formulation $(14,15)$ reduces the background autofluorescence in the channels used for the CFP/YFP FRET; however, it is no longer available from this manufacturer. We list the formulation in Table 3.
} 
5. Inverted tissue culture microscope (Olympus CKX31) equipped with a microinjection system (Femtojet system; Eppendorf).

6. Microinjection needles are pulled using a Sutter P-97 puller (Sutter Instruments) following the manufacturer's protocols. We use borosilicate glass capillary tubes with filament, $10 \mathrm{~cm}$ length with $1.0 \mathrm{~mm}$ outer diameter and $0.58 \mathrm{~mm}$ inner diameter (Sutter Instruments: BF100-58-10). Ramp test should be performed on the puller to determine the proper melting point of the glass.

7. Loading pipette tips (Eppendorf).

8. Multispectral beads for calibrations (Bangs Laboratory, custom design courtesy of Dr. Robert H. Singer; Cat\#: FS02F; Lot\#: 8227; Inventory\#: L070619A).

9. Metamorph ver.7.6 software (Molecular Devices).

10. Light-intensity meter (ThorLabs).

\section{Methods}

\subsection{Dye Labeling of the Multiplex-Compatible Biosensor for Cdc42}

The CBD-MBP is produced in bacteria essentially as detailed elsewhere for the original MeroCBD biosensor (9). For dual-labeling of the CBD-MBP, we first perform the cysteinetargeted site-specific labeling using mero87-iodoacetamide $(2,4)$ at $\mathrm{pH} 7.5$, followed by a change of $\mathrm{pH}$ to an acidic condition ( $\mathrm{pH}$ 5.5) and perform the N-terminal amine-targeted site-specific labeling using Atto-700-NHS-succinimidylester.

1. The mero87 labeling of the CBD-MBP is based on the cysteine-targeted chemistry we used previously (2). We normally use $600-800 \mu \mathrm{l}$ of the CBD-MBP protein stock solution at $200 \mu \mathrm{M}$ in $50 \mathrm{mM} \mathrm{NaH}_{2} \mathrm{PO}_{4}$ at $\mathrm{pH} 7.5$ to take into account the later loss of protein through precipitation at lower $\mathrm{pH}$ conditions. One milligram of Mero87-iodoacetamide is dispersed into $20 \mu \mathrm{l}$ of anhydrous DMSO which will yield a concentration of approximately $40 \mathrm{mM}$ (measure precisely using the extinction coefficient of 135,000 in DMSO at $596 \mathrm{~nm}$ absorption). This stock dye solution is then added into the protein solution to yield a $6: 1$ dye to protein ratio in the final reaction mix. This mixture is quickly vortexed, and put on a rocking mixer for $1 \mathrm{~h}$ at room temperature (see Note 3 ).

2. At the end of $1 \mathrm{~h}$, the reaction is quenched by the addition of $3 \mu \mathrm{l}$ of 2mercaptoethanol, and allowed to further proceed the quenching reaction at room temperature on the rocking mixer for additional $5 \mathrm{~min}$. The mixture is then briefly centrifuged at $20,000 \times g$ for $1 \mathrm{~min}$ at room temperature.

3. For the purification of the labeled material, use a Sephadex G-15 column equilibrated with $50 \mathrm{mM} \mathrm{NaH}_{2} \mathrm{PO}_{4}$ at $\mathrm{pH}$ 7.5.

4. Following a successful labeling of the CBD-MBP, the protein is concentrated using a centrifugal concentrator to the final concentration of $200 \mu \mathrm{M}$, and it can be frozen at $-86^{\circ} \mathrm{C}$ for storage.

5. The N-terminal labeling of the CBD-MBP-mero87 requires the $\mathrm{pH}$ of the buffer to be reduced to 5.5, rendering the intra-chain lysines nonreactive. At this condition,

\footnotetext{
${ }^{2}$ The production of oxygen radicals during the fluorescence excitation of the fluorophores must be rigorously controlled, particularly when having two biosensor systems in a single living cell, as this necessitates a greater dose of exposure to the fluorescence excitation light. We do this through pretreatment of the imaging media with Ar gas followed by the Oxyfluor reagent treatment.

3 There could be batch-to-batch variations in the reactivity of the dye. The proposed 6:1 dye to protein ratio and the reaction time of 1 $\mathrm{h}$ at $24^{\circ} \mathrm{C}$ should be optimized as required to achieve $95-100 \%$ efficiency of labeling.
} 
the stability of the protein is slightly compromised and we have found that $3 \mathrm{~h}$ is the maximum we can maintain the protein at this reduced $\mathrm{pH}$ and still obtain workable yields. To exchange the $\mathrm{pH}$, dialyze $600-800 \mu \mathrm{l}$ of the mero87-labeled CBD-MBP against a $4 \mathrm{~L}$ volume of $50 \mathrm{mM} \mathrm{NaH}_{2} \mathrm{PO}_{4}$ at $\mathrm{pH} 5.5$ for $1 \mathrm{~h}$ at $24^{\circ} \mathrm{C}$ (see Note 4).

6. Immediately following the dialysis, protein concentration is measured spectrophotometrically and the protein is then concentrated as required to $200 \mu \mathrm{M}$ using a centrifugal concentrator.

7. One milligram of Atto 700 NHS is first dissolved in $40 \mu$ of anhydrous DMSO and the concentration is measured spectrophotometrically based on the extinction coefficient provided by the manufacturer. Here, we aim at a $20-40 \mathrm{mM}$ stock concentration. The optimal dye to protein ratio should be empirically determined, but a starting point should be approximately 10:1 (see Note 5). Allow the labeling reaction to proceed at $24^{\circ} \mathrm{C}$ for $2 \mathrm{~h}$ on a rocking mixer.

8. At the end of $2 \mathrm{~h}$, quench the reaction by addition of $1 / 3$ reaction volume of $1.5 \mathrm{M}$ hydroxylamine at $\mathrm{pH} 5.5$ and then allow the quenching reaction to proceed for 5 min prior to a brief centrifugation at $20,000 \times g$ for $1 \mathrm{~min}$ at room temperature and purification using a size exclusion column.

9. For the purification of the labeled material, use a Sephadex G-15 column equilibrated with $50 \mathrm{mM} \mathrm{NaH}_{2} \mathrm{PO}_{4}$ at pH 5.5 (see Note 6). Upon collection, the labeled fraction is immediately subjected to dialysis against $50 \mathrm{mM} \mathrm{NaH}_{2} \mathrm{PO}_{4}$ at $\mathrm{pH} 7.5$ at $4^{\circ} \mathrm{C}$ overnight.

10. Following the dialysis, the protein concentration is determined spectrophotometrically. Here, care must be taken to properly account for the multiple labeling effects. The correction factors for the absorption measurements at $280 \mathrm{~nm}$ must first be determined. In order to calculate these, make dilutions of the dyes in $50 \mathrm{mM} \mathrm{NaH}_{2} \mathrm{PO}_{4}$ buffer and measure the spectra from $280 \mathrm{~nm}$ to the dye absorption maxima for both the mero87 and Atto 700. Take the absorption value at $280 \mathrm{~nm}$ from these solutions in the absence of any proteins, and divide that value by the absorption value of the major dye absorbance peak. The resulting correction factor is the percentage absorption of the dye at $280 \mathrm{~nm}$ when the dye-conjugated protein concentration is measured. We have determined that the correction factor at $280 \mathrm{~nm}$ is 0.076 for mero87, and 0.4166 for Atto 700 .

11. Adjust the protein concentration to approximately $50 \mu \mathrm{M}$ and flash freeze $40 \mu \mathrm{l}$ aliquots of CBD-MBP-mero87-Atto700 (Cdc42 biosensor) using liquid $\mathrm{N}_{2}$, and store at $-86^{\circ} \mathrm{C}$.

\subsection{Viral Transduction and Stable tet-OFF Cell Line Production}

1. Plate GP2-293 cells at $8 \times 10^{6}$ cells/plate overnight in a 10 -cm tissue culture dish coated with poly-L-lysine.

2. Prepare transfection mix. The optimal amounts of DNA plasmids for the transfection are $22 \mu \mathrm{g}$ of pRetro-X-tet-OFF and $2 \mu \mathrm{g}$ of pVSVg together with $60 \mu \mathrm{l}$

\footnotetext{
${ }^{4}$ We find it critical that this $\mathrm{pH}$ exchange be a dialysis-based rather than using an ion/salt exchange column. It appears that using these ion/salt exchange columns does not result in a proper protonation of the intrachain lysines in our hands and always results in excessive conjugation of the succinimidylester dye.

${ }^{5}$ We have found that the reactivity of this dye is somewhat low and have therefore required a relatively large dye to protein ratio for the optimal reaction.

${ }^{6}$ The separation of the labeled material from the unlabeled free dye is usually straightforward. Only caution here is a potential overloading of the column resulting in an incomplete separation and smearing.
} 
of Lipofectamine 2000 reagent in a total transfection mix volume of $1 \mathrm{ml}$ following the manufacturer's instructions. Allow complexes to form for approximately 20 $\min$ at $24^{\circ} \mathrm{C}$ (see Note 7 ).

3. Add the mix dropwise to the cell monolayer to result in a total volume of $5 \mathrm{ml}$.

4. Exchange medium for $7 \mathrm{ml}$ of fresh growth medium containing 5\% FCS and normal antibiotic concentrations $24 \mathrm{~h}$ posttransfection, and transfer plates to a $32^{\circ} \mathrm{C}$ incubator.

5. Collect viral supernatant $48 \mathrm{~h}$ following the medium exchange. Concentrate the viral supernatant using a Retro-X concentrator following the manufacturer's protocols to a 7:1 ratio. Store at $-80^{\circ} \mathrm{C}$ for later use (see Note 8 ).

6. Plate MEFs (or the cells of interest) at $1 \times 10^{5}$ cells/plate overnight in a $10-\mathrm{cm}$ tissue culture dish.

7. Add supernatant stock ( $0.5 \mathrm{ml}$ of $7: 1$ concentrated supernatant) together with $8 \mu \mathrm{g} /$ $\mathrm{ml}$ polybrene in a total medium volume of $4 \mathrm{ml}$. We normally infect four consecutive times, $12 \mathrm{~h}$ apart, before starting the selection.

8. Select for the stable incorporation of the tet-OFF tetracyclin trans-activator (tTA) using G-418 at the final concentration of $1 \mathrm{mg} / \mathrm{ml}$.

\subsection{Viral Transduction and Stable Biosensor Cell Line Production}

1. Perform production of the virus containing the biosensor expression construct as described in Subheading 3.2, steps 1 through 5.

2. Perform transduction as described in step 7 of Subheading 3.2. However, it is necessary to monitor the cell fluorescence every $24 \mathrm{~h}$ (every second consecutive transduction) to ascertain that a sufficient level of biosensor expression is achieved prior to repressing the biosensor expression and then initiating the selection process.

3. The selection for stable incorporation of the biosensor must be performed in a stepwise manner, by gradually increasing the puromycin or hygromycin concentration. We routinely start at $1 \mu \mathrm{g} / \mathrm{ml}$ for the puromycin selection and $10 \mu \mathrm{g} /$ $\mathrm{ml}$ for the hygromycin selection. The final concentrations for the stable selection for MEFs are $10 \mu \mathrm{g} / \mathrm{ml}$ puromycin and $50 \mu \mathrm{g} / \mathrm{ml}$ hygromycin (see Note 9 ).

4. Once the stable, inducible cell lines are established, cells are FACS sorted based on the fluorescence intensity of the expressed biosensors to obtain populations of low, medium, and high expressors.

5. The induction procedure for the biosensors is to remove the doxycyclin from the growth medium. Cells cultured in medium containing doxycyclin are detached when subconfluent, pelleted at $300 \times g$, and washed once with fresh medium without doxycyclin. The cells are then replated onto fresh $10-\mathrm{cm}$ tissue culture dishes at $5 \times$ $10^{4}$ cells/plate without doxycyclin. Imaging is typically performed $48-72 \mathrm{~h}$ postinduction depending on the efficiency of the induction in different cell types (see Note 10).

\footnotetext{
${ }^{7}$ Transfection is best performed by reducing the DNA suspension media and Lipofectamine 2000 suspension volumes to $500 \mu$ leach. Here, it is critical to remove any antibiotics from the media during transfection.

${ }^{8}$ Because VSVg is toxic, it has been possible to reliably collect only one round of the supernatant in our hands. Therefore, we normally produce multiple tissue culture dishes of viral supernatant, pool them, and then concentrate the virus at a ratio of 7:1.

${ }^{9}$ We usually transduce 4-6 consecutive times, and then repress the biosensor expression by applying doxycycline at the final concentration of $2 \mu \mathrm{g} / \mathrm{ml}$ prior to starting the selection process.
} 


\subsection{A Typical Procedure and Key Considerations for the Single-Mode Imaging of the Rho GTPase Biosensor}

1. Induced cells are plated onto sterile, precleaned, $25-\mathrm{mm}$ round coverslips at $4.5 \times$ $10^{4}$ cells/coverslip in normal growth medium. We find it convenient to place the coverslips in 6-well plates. The coverslips are usually treated with fibronectin prior to plating.

2. Allowed cells to attach and spread for approximately $2-3 \mathrm{~h}$ prior to imaging. The imaging medium used is Ham's F-12K without phenol-red (Biosource).

3. Assemble the imaging chamber (see Fig. 5) (see Note 11).

4. For the sequential acquisition mode, follow the flow diagram of Fig. 6a. Control the image acquisition using the Metamorph ver.7.6 software and use the macro programs that automate the majority of acquisition functions together with the multidimensional acquisition application. In order to ascertain the extent of potential motion artifacts, we acquire first a CFP image followed by the FRET image, and then one additional CFP image at every acquisition time point. The ratio between the first CFP image and the last CFP image constitutes the extent of motion artifacts at each time point.

5. For the simultaneous acquisition mode, follow the flow diagram of Fig. 6b. As in the sequential acquisition mode, use the Metamorph software with the automation macro programs.

Concerning beam-splitting at the side port for CFP and FRET on two cameras, if a beamsplitter that does not require a relay lens cannot be placed in the focused distance of the tube lens, there are two possible solutions: (a) use the commercially available Dual-View/DualCam module (Optical Insights) which would reduce the light throughput due to inclusions of additional relay lens components (16-25\% loss as measured in our hands) or (b) remove the tube lens from the microscope and perform all of the beam-splitting and filtering in the infinity space outside of the microscope body and then bring to focus the two channels at some distance away from the final beam-splitter. The second solution requires additional thoughts, in that not all manufacturers fully correct the objective lens and some rely on the tube lens to perform a portion of the lateral chromatic correction (excepting the Nikon CF optics). Therefore, if more than one tube lens is used or if nonoriginal equipment manufacturer lenses are used, this issue will become problematic.

One of the key points is to choose the dual-camera system so that the cameras are identical. We carefully test the CCD readout noise characteristics of two identical camera units and select based on similar noise characteristics. Typically, readout noise of less than six electrons at $1 \times 1$ binning is desired and the two CCDs should have near identical noise characteristics. The beam-splitter that reflects the image onto the second CCD camera requires some consideration. The ideal approach would be to place the dichromatic mirror directly within the emission optical train without additional relay lenses. There are several approaches to achieving this. If bottom and side ports of the microscope can be dedicated to the CFP/YFP FRET acquisition modality, the simplest solution is to replace the internal side

\footnotetext{
${ }^{10}$ We have seen the timing for the optimal imaging postinduction to vary depending on the particular cell type; for example, MEFs appear to be optimal at $48 \mathrm{~h}$, while the rat mammary carcinoma MtLn3 cell line requires $72 \mathrm{~h}$ for an optimal induction. It is possible to slightly boost the total expression level of the biosensor by simply trypsinizing and replating directly back onto the same culture dish at 24-h intervals, though we find this to be rarely necessary.

${ }^{11}$ For stimulation experiments, the glass coverslip comprising the top part of the heated chamber is excluded during the experiment. In this case, $25 \mathrm{mM}$ HEPES buffer should be added to the medium, and stimulation experiments should be designed in such a way to ensure a good mixing upon addition of the stimulant. We have found that having $500 \mu \mathrm{l}$ of medium in this open chamber and an addition of another $500 \mathrm{~L}$ with the stimulant at $2 \times$ concentration works effectively.
} 
port prism to an appropriate long-pass mirror and then attach the two cameras par-focal at the two ports, placing appropriate emission bandpass filters in front of each camera. If only one camera port is available, it is usually possible to attach a beam-splitter module within the focused distance of the optical train without the need for an additional relay lens by using a dual-camera module such as the U-DPCAD from Olympus for the IX2 series of microscopes. For the image acquisition, we take simultaneously FRET and CFP emissions upon a single CFP excitation using the two-camera setup at each time point during a timelapse experiment. Following image acquisitions, it is necessary to take a calibration image set using a coverslip with mounted multispectral beads. Because the mounting of cameras and the dichromatic mirrors are never perfect and are susceptible to fluctuations, it is necessary to correct for any potential misalignment between the FRET and CFP channels prior to ratiometric calculations. In order to achieve the required pixel-by-pixel register, the calibration image set is used to obtain the nonlinear field morphing parameters using a computer algorithm available elsewhere (9). Using this approach, it is possible to register two images to an accuracy of one-twentieth of a pixel, allowing for corrections of chromatic aberrations as well as translational and shear effects within the FRET/CFP image set.

\subsection{A Typical Procedure and Key Considerations for the Multiplex-Mode Imaging of Rho GTPase Biosensors}

1. Plate induced cells onto fibronectin-coated $25-\mathrm{mm}$ round coverslips as described in step 1 of Subheading 3.4. The cells to be imaged must be healthy and should have been passaged at optimal confluence. The microinjection of the $\mathrm{Cdc} 42$ biosensor is performed $\sim 4 \mathrm{~h}$ postplating.

2. Thaw a frozen $40-\mu \mathrm{l}$ aliquot of the Cdc42 biosensor over ice, adjust to $50 \mu \mathrm{M}$ using $50 \mathrm{mM} \mathrm{NaH}_{2} \mathrm{PO}_{4} \mathrm{pH} \mathrm{7.5}$, and centrifuge at $15,000 \times g$ at $4^{\circ} \mathrm{C}$ for $20 \mathrm{~min}$. Carefully transfer the supernatant to a fresh tube, wrapped in aluminum foil and keep on ice until microinjection.

3. Transfer coverslips with cells from 6-well plates to a 35-mm round tissue culture dish with approximately $4 \mathrm{ml}$ of regular growth medium. This is mounted atop the inverted tissue culture microscope equipped with a microinjection system.

4. Using a loading pipette tip back-fill $5 \mu \mathrm{l}$ of the $\mathrm{Cdc} 42$ biosensor solution into the pulled needles and attached a needle tip to the microinjection system. Typically, we inject 20-40 cells per coverslip in approximately $10 \mathrm{~min}$ at ambient conditions. MEFs appear to lose integrity much past this 10 min window and this affects the survival rate following the injection and recovery. Cells are allowed to recover for $30 \mathrm{~min}$ in the incubator prior to imaging.

5. Assemble the imaging chamber (see Fig. 5) (see Note 11).

6. Follow the flow diagram of Fig. 7. We use the Metamorph software with the automation macro programs. The image acquisition sequence is such that we first autofocus to find the focused plane, followed by CFP/YFP FRET acquisition using the side-mounted two-camera setup, then acquire the mero87/Atto700 using the bottom-mounted two-camera setup, and then acquire a DIC image using one of the channels of the bottom-mounted cameras. Here, we need to place a linear polarizer in and out of the light path for the DIC imaging; therefore we have designed a filterwheel within the focused distance of the bottom port prior to the beam-splitter (see Fig. 4). For the beam-splitter, we use the DualCam module (Optical Insights) with the appropriate set of filters (Table 2). Because of the complexity of imaging paths (see Fig. 4), we have opted for using this beam splitting solution even with the loss of light associated with the internal relay optics within the DualCam. In doing so, we have retained the original lateral chromatic correction characteristics 
of the tube lens and enough distance to place a single filterwheel within the optical path, greatly expanding the versatility of the imaging system. Again, it is possible to achieve a system that is more light efficient through the placement of a tube lens at some distant position away from the original design of the microscope.

Similar image processing considerations as in the single-mode, simultaneous imaging described in Subheading 3.4 apply. The primary concern is therefore the channel-to-channel alignment on a pixel-by-pixel basis prior to ratiometric calculations. Although we maintain the types of CCDs identical on all four channels, we have noticed significant magnification differences between the side port versus the bottom port of the microscope. This should also be corrected using the morphing-based image registration approaches described elsewhere (9).

Since the excitations of mero87 and Atto700 are achieved using two different light sources, the light intensity of each excitation source must be carefully monitored. We use a lightintensity meter to measure the milliWatt power of light at each excitation wavelength at the specimen plane prior to imaging. Usually, we measure approximately $0.5 \mathrm{~mW}$ of light power at each excitation wavelength, $\pm 5 \%$. If the light intensities are significantly different, neutral density filters must be used to adjust the relative exposure intensities at the two cameras.

Imaging conditions are carefully selected so as to minimize the production of the reactive oxygen species. It appears that cells tolerate much better longer exposure times at low light intensities compared to a short exposure at a high excitation light intensity. To achieve this, the multiplex imaging system (see Fig. 4 and Table 2) has been optimized to deliver a low intensity excitation through a combination of the neutral density filters and the 94:6 reflective mirror in the main turret. The use of this mirror allows one to sidestep the need for a custom-designed dichromatic mirror and then use the bandpass filters that are designed very close in wavelength separation to one another. This approach enables the system to: (1) tightly pack the bandpass filters allowing separation of more colors within the visible spectrum and (2) separate multiple fluorescence wavelengths at a minimal loss of light compared to using the traditional dichromatic mirrors. Using this system, we routinely achieve $\sim 1,000 \mathrm{~ms}$ exposure at ND1.0 (10\% incident light) to fill approximately $80 \%$ of the dynamic range of the cameras.

\section{Acknowledgments}

This work was funded by GM093121 (D.S. and L.H.) and "Sinsheimer Foundation Young Investigator Award" (L.H.).

\section{References}

1. Kraynov VS, Chamberlain C, Bokoch GM, Schwartz MA, Slabaugh S, Hahn KM. Localized Rac activation dynamics visualized in living cells. Science. 2000; 290:333-337. [PubMed: 11030651]

2. Nalbant P, Hodgson L, Kraynov V, Toutchkine A, Hahn KM. Activation of endogenous Cdc42 visualized in living cells. Science. 2004; 305:1615-1619. [PubMed: 15361624]

3. Pertz O, Hodgson L, Klemke RL, Hahn KM. Spatiotemporal dynamics of RhoA activity in migrating cells. Nature. 2006; 440:1069-1072. [PubMed: 16547516]

4. Machacek M, Hodgson L, Welch C, Elliott H, Pertz O, Nalbant P, Abell A, Johnson GL, Hahn KM, Danuser G. Coordination of Rho GTPase activities during cell protrusion. Nature. 2009; 461:99_ 103. [PubMed: 19693013]

5. Seth A, Otomo T, Yin HL, Rosen MK. Rational design of genetically encoded fluorescence resonance energy transfer-based sensors of cellular Cdc42 signaling. Biochemistry. 2003; 42:39974008. [PubMed: 12680752] 
6. Ridley AJ, Hall A. The small GTP-binding protein rho regulates the assembly of focal adhesions and actin stress fibers in response to growth factors. Cell. 1992; 70:389-399. [PubMed: 1643657]

7. Ridley AJ, Paterson HF, Johnston CL, Diekmann D, Hall A. The small GTP-binding protein rac regulates growth factor-induced membrane ruffling. Cell. 1992; 70:401-410. [PubMed: 1643658]

8. Garrett SC, Hodgson L, Rybin A, Toutchkine A, Hahn KM, Lawrence DS, Bresnick AR. A biosensor of S100A4 metastasis factor activation: inhibitor screening and cellular activation dynamics. Biochemistry. 2008; 47:986-996. [PubMed: 18154362]

9. Hodgson, L.; Shen, F.; Hahn, KM. Biosensors for characterizing the dynamics of rho family GTPases in living cells. In: Bonifacino, JS.; Dasso, M.; Harford, JB.; Lippincott-Schwartz, J.; Yamada, KM., editors. Current Protocols in Cell Biology. Vol. Unit 14.11. Wiley; New York: 2010.

10. Gossen M, Bujard H. Tight control of gene expression in mammalian cells by tetracyclineresponsive promoters. Proc Natl Acad Sci USA. 1992; 89:5547-5551. [PubMed: 1319065]

11. Hodgson L, Pertz O, Hahn KM. Design and optimization of genetically encoded fluorescent biosensors: GTPase biosensors. Methods Cell Biol. 2008; 85:63-81. [PubMed: 18155459]

12. Ouyang M, Huang H, Shaner NC, Remacle AG, Shiryaev SA, Strongin AY, Tsien RY, Wang Y. Simultaneous visualization of protumorigenic Src and MT1-MMP activities with fluorescence resonance energy transfer. Cancer Res. 2010; 70:2204-2212. [PubMed: 20197470]

13. Toutchkine A, Nguyen DV, Hahn KM. Merocyanine dyes with improved photostability. Org Lett. 2007; 9:2775-2777. [PubMed: 17583344]

14. Kaighn, ME. Tissue Culture Methods and Applications. Academy Press; New York: 1973.

15. Robey PG, Termine JD. Human bone cells in vitro. Calcif Tissue Int. 1985; 37:453-460. [PubMed: 2998572] 


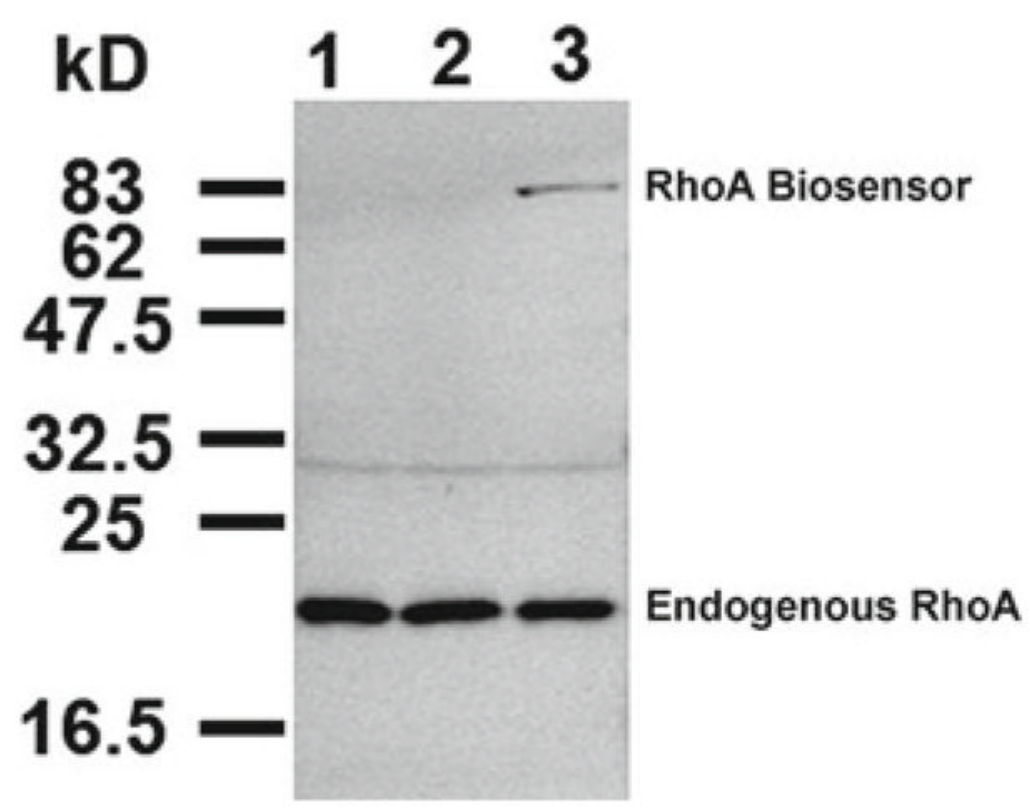

Fig. 1.

Expression levels of the genetically encoded single-chain RhoA biosensor in MEFs, taken from ref. 4. Lane 1: doxycyclin at $1 \mu \mathrm{g} / \mathrm{ml}$; Lane 2: doxycyclin at $0.1 \mu \mathrm{g} / \mathrm{ml}$; and Lane 3: no doxycyclin. The expression level of the biosensor is approximately $20 \%$ of the endogenous RhoA under these conditions (4). 


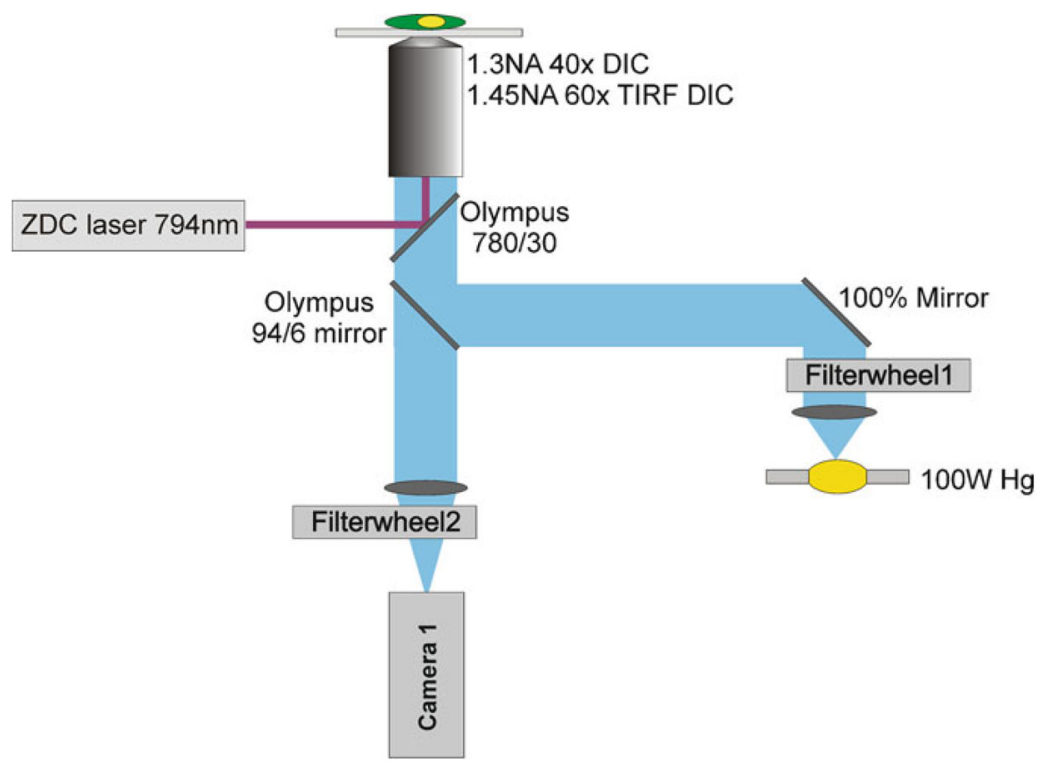

Fig. 2.

The light path diagram for the single-camera, single-biosensor mode imaging. The list of bandpass filters and dichromatic mirrors required in this setup is shown in Table 1. 


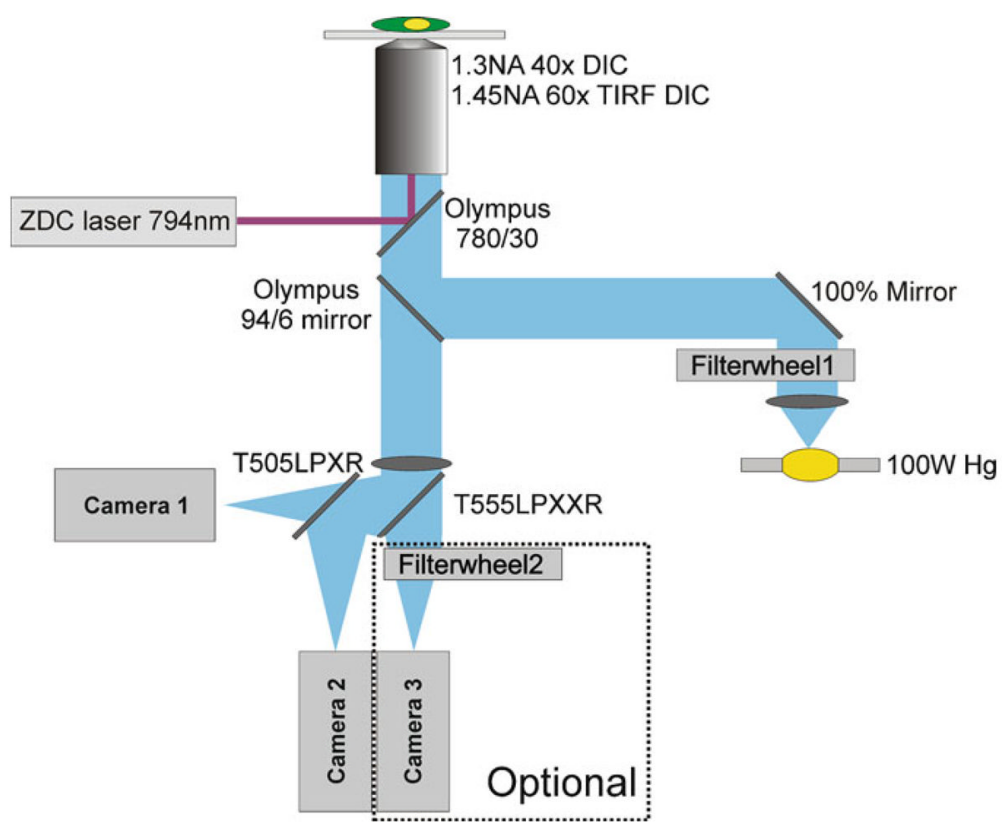

Fig. 3.

The light path diagram for the dual-camera, single-biosensor mode imaging. Optional third camera path is also shown. The third camera will enable acquisitions of DIC and any other red and far-red fluorescence beyond the cut-off point of the long-pass filter to be placed in the optical path. The list of bandpass filters and dichromatic mirrors required in this setup is shown in Table 1. 


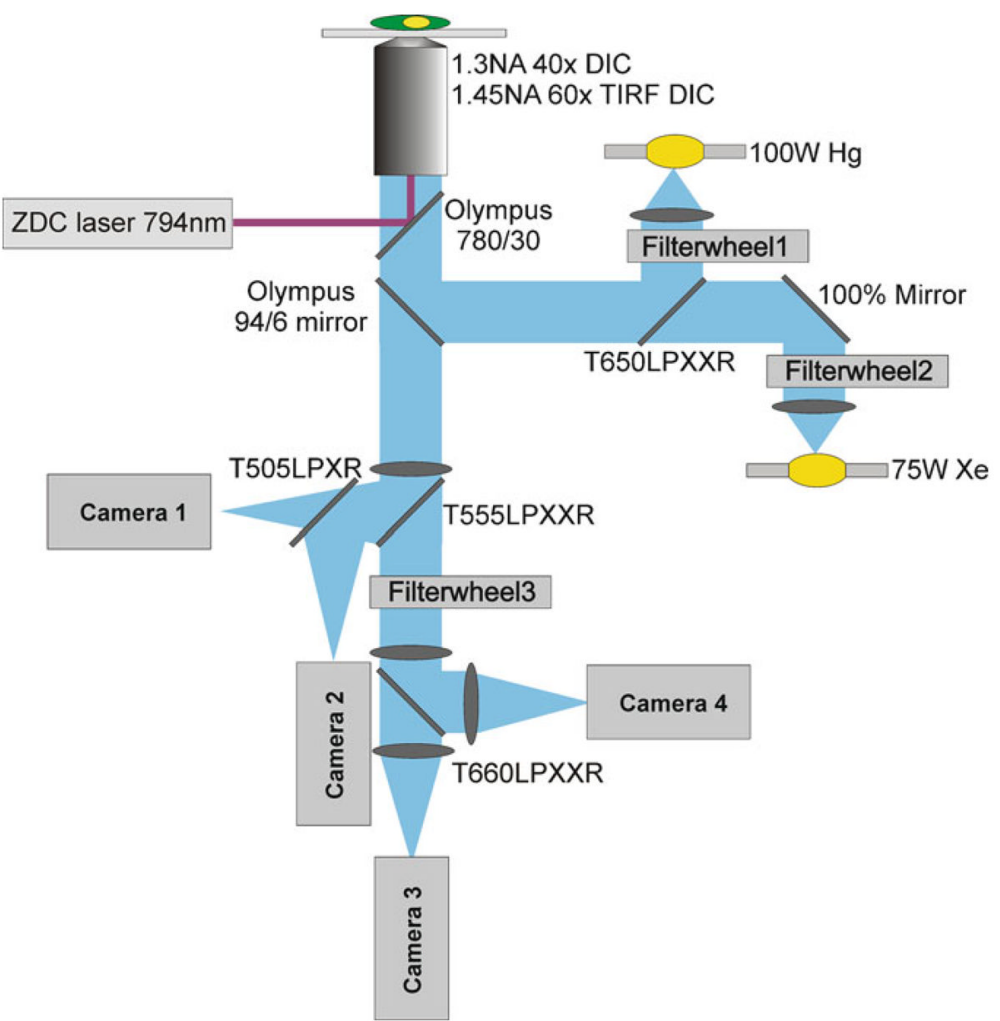

Fig. 4.

The light path diagram for the four-channel, dual-biosensor mode imaging. The list of bandpass filters and dichromatic mirrors required in this setup is shown in Table 2. 
a

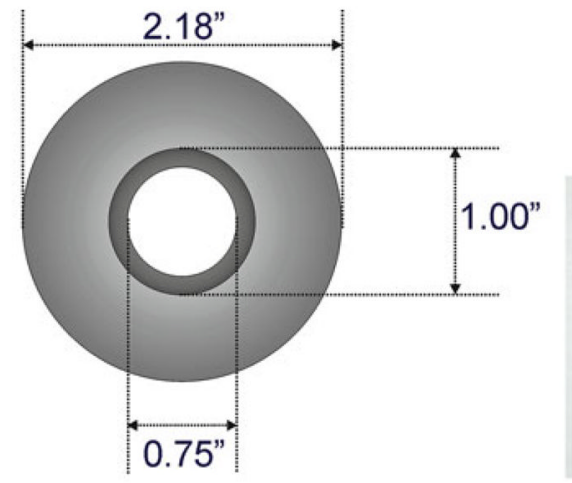

b

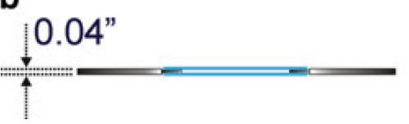

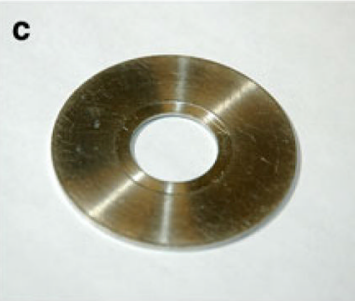

Fig. 5.

Design of the custom imaging chamber. (a) Top view of the chamber with appropriate measurements. (b) Side view of the chamber. The two 25-mm round coverslips are assembled in a sandwich-like manner using silicone vacuum grease. Cells are cultured onto these coverslips and the small void created by the chamber and the two coverslips contains the imaging medium (approximately $280 \mu \mathrm{l}$ ). (c) Photograph of an actual chamber. The chamber assembly was designed to fit into the QE-2 heated stage assembly of Warner Instruments. 


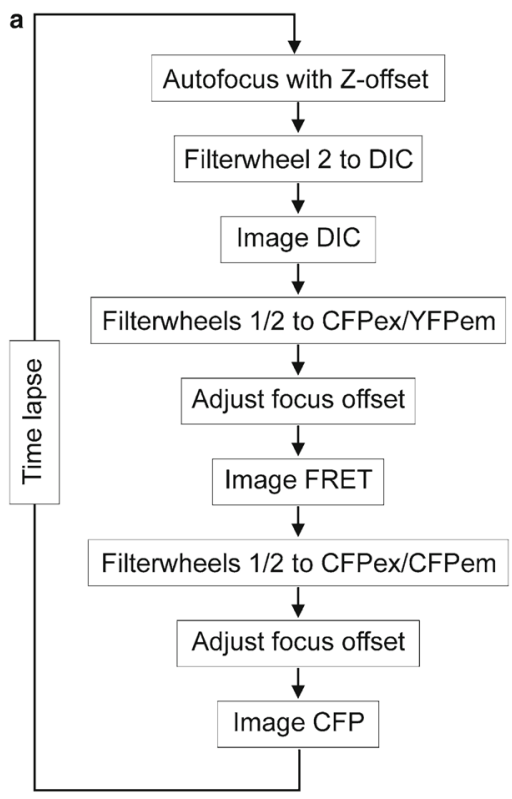

b

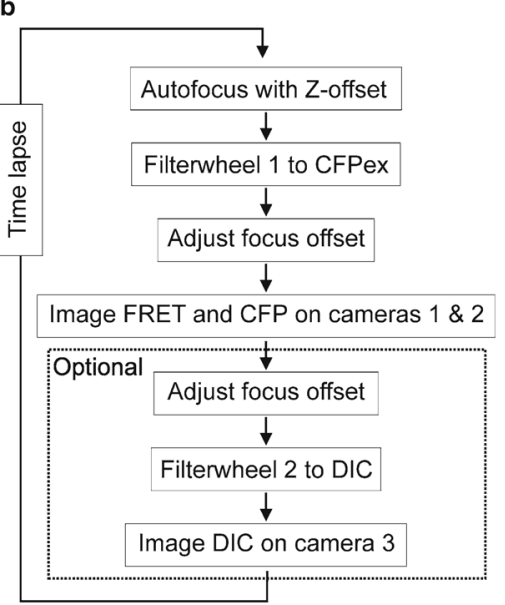

Fig. 6.

(a) Flow diagram for the single-camera, single-biosensor mode imaging. The focus offsets must be individually determined empirically for each cell type and wavelength. (b) Flow diagram for the dual-camera, single-biosensor mode imaging. The optional DIC acquisition using a third camera is also shown. 


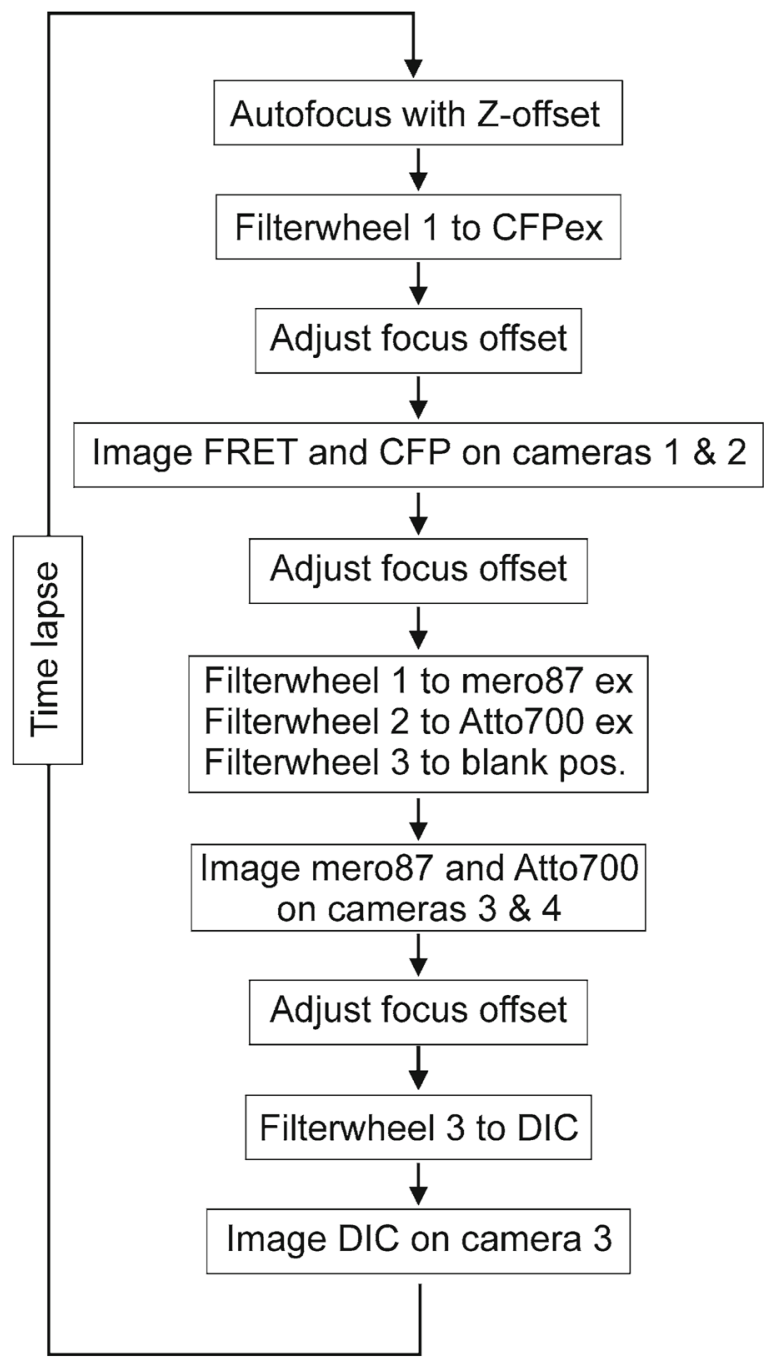

Fig. 7.

Flow diagram for the four-channel, dual-biosensor mode imaging. 
Table 1

List of bandpass filters and dichromatic mirrors required for the single-biosensor mode imaging

\begin{tabular}{lll|}
\hline \multicolumn{2}{l}{ Single-camera/single-mode imaging } \\
Bandpass filters & & \\
ET436/20X & CFP excitation & Filterwheel 1 \\
ET500/20X & YFP excitation & Filterwheel 1 \\
ET480/40M & CFP emission & Filterwheel 2 \\
ET535/30M & YFP (FRET) emission & Filterwheel 2 \\
\hline Dichromatic mirrors & \\
94:6 mirror & All fluororphores & Main turret \\
\hline Dual-camera/single-mode imaging (optional third camera) \\
\hline Bandpass filters & & \\
ET436/20X & CFP excitation & Filterwheel 1 \\
ET500/20X & YFP excitation & Filterwheel 1 \\
ET480/40M & CFP emission & At camera 2 \\
ET535/30M & YFP(FRET) emission & At camera 1 \\
Linear polarizer & DIC camera 3 & Filterwheel 2 \\
\hline Dichromatic mirrors & \\
94:6 mirror & All fluororphores & Main turret \\
T555LPXXR & CFP/FRET to side port & At side port prism \\
T505LPXR & Separate CFP/FRET & Side port beam-splitter \\
\hline
\end{tabular}

The bandpass filters and the dichromatic mirrors are from Chroma Technology. The 94:6 mirror is from Olympus. The linear polarizer is from Chroma Technology. All filters and mirrors are antireflection coated 
Table 2

List of bandpass filters and dichromatic mirrors required for the dual-biosensor mode imaging

\begin{tabular}{|lll|}
\hline Four-channels/dual-mode imaging & \\
Bandpass filters & & \\
ET436/20X & CFP excitation & Filterwheel 1 \\
ET500/20X & YFP excitation & Filterwheel 1 \\
ET480/40M & CFP emission & At camera 2 \\
ET535/30M & YFP(FRET) emission & At camera 1 \\
Linear polarizer & DIC camera 4 & Filterwheel 3 \\
FF585/29 & mero87 excitation & Filterwheel 1 \\
FF684/24 & Atto700 excitation & Filterwheel 2 \\
FF628/32 & mero87 emission & At camera 4 \\
FF794/160 & Atto700 emission & At camera 3 \\
\hline Dichromatic mirrors & \\
94:6 mirror & All fluororphores & Main turret \\
T650LPXXR & Combine Xe and Hg & Arc lamp combiner \\
T555LPXXR & CFP/FRET to side port & At side port prism \\
T505LPXR & Separate CFP/FRET & Side port beam-splitter \\
T660LPXXR & Separate mero87/Atto700 & Side port beam-splitter \\
\hline
\end{tabular}

The bandpass filters and the dichromatic mirrors starting with the "ET" and "T" specifications, respectively, are from Chroma Technology. The bandpass filters with the "FF" specifications are from Semrock. The 94:6 mirror is from Olympus. The linear polarizer is from Chroma Technology. All filters and mirrors are antireflection coated 
Table 3

Formulation of the Ham's F-12K medium without phenol-red and without glutamine, $\mathrm{pH}$ 7.4-7.5

\begin{tabular}{|llll|}
\hline Inorganic salts & $\mathbf{m g} / \mathbf{L}$ & Other compounds & $\mathbf{m g} / \mathbf{L}$ \\
\hline $\mathrm{NaCl}$ & 7,530 & Glucose & 1,260 \\
$\mathrm{KCl}$ & 285 & Linoleic acid & 0 \\
$\mathrm{MgCl}_{2}-6 \mathrm{H}_{2} \mathrm{O}$ & 106 & Hypoxanthine-Na & 4 \\
$\mathrm{MgSO}_{4}-7 \mathrm{H}_{2} \mathrm{O}$ & 393 & Phenol-red & 0 \\
$\mathrm{CaCl}_{2}$ & 135 & Putresine-HCl & 0.3 \\
$\mathrm{Na}_{2} \mathrm{HPO}_{4}-7 \mathrm{H}_{2} \mathrm{O}$ & 218 & Sodium pyruvate & 220 \\
$\mathrm{KH}_{2} \mathrm{PO}_{4}$ & 59 & Thymidine & 0.7 \\
$\mathrm{NaHCO}_{3}$ & 2,500 & & \\
$\mathrm{FeSO}_{4}-7 \mathrm{H}_{2} \mathrm{O}$ & 0.8 & & \\
$\mathrm{CuSO}_{4}-5 \mathrm{H}_{2} \mathrm{O}$ & 0.002 & & \\
$\mathrm{ZnSO}_{4}-7 \mathrm{H}_{2} \mathrm{O}$ & 0.14 & & \\
\hline
\end{tabular}

\begin{tabular}{|llll|}
\hline Amino acids & $\mathbf{m g} / \mathbf{L}$ & Vitamins & $\mathbf{m g} / \mathbf{L}$ \\
\hline L-Alanine & 17.8 & L-Ascorbic acid & 0 \\
L-Arginine-HCl & 421.3 & Biotin & 0.07 \\
L-Asparagine- $\mathrm{H}_{2} \mathrm{O}$ & 30 & D-Calcium pantothenate & 0.48 \\
L-Aspartic acid & 26.6 & Choline chloride & 13.96 \\
L-Cysteine-HCl- $\mathrm{H}_{2} \mathrm{O}$ & 70.04 & Cyanocobalamin & 1.36 \\
L-Cystine & 0 & Folic acid & 1.32 \\
L-Glutamic acid & 29.4 & Inositol & 18 \\
Glycine & 15 & Nicotinamide & 0.04 \\
L-Histidine-HCl- $\mathrm{H}_{2} \mathrm{O}$ & 41.9 & Pyridoxine-HCl & 0.06 \\
L-Isoleucine & 7.9 & Riboflavin & 0.04 \\
L-Leucine & 26.2 & Thiamine-HCl & 0.21 \\
L-Lysine-HCl & 73.1 & DL-Thioctic acid & 0.21 \\
L-Methionine & 8.9 & & \\
L-Phenylalanine & 9.9 & & \\
L-Proline & 69.1 & & \\
L-Serine & 21 & & \\
L-Threonine & 23.8 & & \\
L-Tryptophan & 4.1 & & \\
L-Tyrosine & 10.9 & & \\
L-Valine & 23.4 & & \\
\hline
\end{tabular}

